\title{
Differentially expressed plasma microRNAs in premature ovarian failure patients and the potential regulatory function of mir-23a in granulosa cell apoptosis
}

\author{
Xiaokui Yang, Ying Zhou, Sha Peng, Liang Wu ${ }^{1,2}$, Hai-Yan Lin ${ }^{1}$, Shuyu Wang and Hongmei Wang ${ }^{1}$ \\ Department of Human Reproductive Medicine, Beijing Obstetrics and Gynecology Hospital, Capital Medical \\ University, Beijing 100006, China, ${ }^{1}$ State Key Laboratory of Reproductive Biology, Institute of Zoology, Chinese \\ Academy of Sciences, 1 Beichen West Road, Chaoyang District, Beijing 100101, China and ${ }^{2}$ Graduate School of the \\ Chinese Academy of Sciences, Beijing 100039, China
}

Correspondences should be addressed to H Wang; Email: wanghm@ioz.ac.cn, S Wang; Email: yushu572000@yahoo.com.cn

$\mathrm{X}$ Yang, $\mathrm{Y}$ Zhou and S Peng contributed equally to this work

\begin{abstract}
Recent studies implicate the regulatory function of microRNAs (miRNAs) in oocyte maturation and ovarian follicular development. Differentially expressed miRNAs are found in the plasma of premature ovarian failure (POF) patients and normal cycling women. In this study, miRNA-regulated signaling pathways and related genes were described using Gene Ontology (GO) analysis and Kyoto Encyclopedia of Genes and Genomes pathway analysis. The effect of mir-23a on granulosa cell apoptosis was also studied by examining the protein expression of X-linked inhibitor of apoptosis protein (XIAP) and caspase-3, followed by subsequent counting of apoptotic cells after Hoechst 33258 staining. Both GO analysis and pathway analysis suggested that many signaling pathways, including the AKT signaling pathway, steroid hormone receptor signaling pathways, and others, were regulated by this group of differentially expressed miRNAs. A decrease in XIAP expression (mRNA and protein level) and caspase-3 protein levels and an increase in cleaved caspase-3 protein were observed in human ovarian granulosa cells transfected with pre-mir-23a, along with an increased occurrence of apoptosis. In conclusion, differentially expressed miRNAs in the plasma of POF patients may have regulatory effects on proliferation and apoptosis of granulosa cells by affecting different signaling pathways. Mir-23a may play important roles in regulating apoptosis via decreasing XIAP expression in human ovarian granulosa cells.
\end{abstract}

Reproduction (2012) $\mathbf{1 4 4} 235-244$

\section{Introduction}

Premature ovarian failure (POF) is an ovarian disorder of multifactorial origin defined as the occurrence of amenorrhea, hypergonadotropism, and hypoestrogenism in women under the age of 40 years (Beck-Peccoz \& Persani 2006). The incidence is one in 10000 women at the age of 20, one in 1000 at the age of 30, and one in 100 by the age of 40 . POF can be sporadic or familial (4-33\%; Conway 1996, van Kasteren et al. 1999, Vegetti et al. 2000). Aside from its associated fertility problems, POF is a serious endocrine disorder and, if left untreated, can induce a twofold age-specific increase in mortality due to an increased incidence of cardiovascular disease, stroke, and osteoporosis (Snowdon et al. 1989). To better understand the pathogenesis of POF, it is necessary to remember that $<500(0.007 \%)$ of a woman's original seven million oocytes are released during her entire reproductive life, and the rest die during the process of folliculogenesis. Therefore, POF may result from either a reduced number of follicles formed during ovarian development or an increased rate of follicle loss. Studies have demonstrated that various factors, such as GNRH (Billig et al. 1994, Andreu et al. 1998), androgens (Billig et al. 1993), and Nodal (Wang et al. 2006), can induce apoptosis in ovarian granulosa cells and result in follicle loss.

MicroRNAs (miRNAs) are noncoding, single-stranded small RNAs of $\sim 22-24$ nucleotides (nt) that constitute a novel class of gene regulators. The primary miRNA transcript, namely pri-miRNAs, is several kilobases long and undergoes substantial processing in the nucleus, resulting in the generation of a 70- to 90-nt stem-loop precursor miRNA (pre-miRNA). After subsequent processing in the cytoplasm by Dicer, a double-stranded miRNA duplex, which contains 2 nt-long $3^{\prime}$ overhangs that will unwind and form a single-stranded mature miRNA, is generated (Bernstein et al. 2001, Bernstein et al. 2003, Paroo et al. 2007). The mature miRNAs 
repress translation or assist in mRNA degradation in a sequence-specific manner (Ambros 2004, Bartel 2004, Zamore \& Haley 2005). In this way, miRNAs influence various cellular activities including cell proliferation, differentiation, and apoptosis under normal and diseased conditions.

Evidence generated in mouse studies suggests a regulatory function of miRNAs in oocyte maturation and ovarian follicular development (Murchison et al. 2007, Tang et al. 2007). Otsuka et al. (2008) found that Dicer 1 deficiency results in female infertility, which was caused by corpus luteum $(\mathrm{CL})$ insufficiency and resulted at least in part from the impaired growth of new capillary vessels in the ovary. Furthermore, impaired CL angiogenesis in Dicer ${ }^{d / d}$ mice was associated with a lack of miR17-5p and let-7b. Hong et al. (2008) showed that Dicer1 $^{f l / f l}$, antiMüllerian hormone receptor Amhr2 $2^{\mathrm{Cre} /+}$ female mice, where Dicer 1 was selectively knocked out in Müllerian duct derivatives (i.e. the oviduct, uterus, and cervix) and in the granulosa cells of secondary and small antral follicles (Hong et al. 2008), showed a decreased ovulation rate and decreased ovary weights compared with wild-type controls (Hong et al. 2008). Choi et al. (2007) identified 177 miRNAs in the newborn mouse ovary and found that four miRNAs were downregulated approximately twofold in the mouse with a knockdown in an ovarian homeobox gene, a transcription factor necessary for oocyte differentiation. In 2006, Kim et al. (2006) identified 58 miRNAs in the pig genome and confirmed the expression of two of these miRNAs in the porcine ovary using northern blot analysis. Ro et al. (2007) identified 122 miRNAs in the ovaries of 2-week-old and adult mice. Fiedler et al. (2008) identified 13 differentially expressed miRNAs in mouse granulosa cells before and $4 \mathrm{~h}$ after human chorionic gonadotropin (hCG) treatment and their further investigation indicated that mir-21 can block apoptosis of mouse periovulatory granulosa cells.

In 2008, Chim et al. (2008) first reported the existence of miRNAs in maternal plasma. Since then, miRNAs have been detected in several kinds of body fluids including plasma, serum, and urine (Gilad et al. 2008). Plasma miRNAs have become promising potential biomarkers for a series of cancers and other diseases such as hepatocellular carcinoma (Yamamoto et al. 2009), gastric cancer (Tsujiura et al. 2010), prostate cancer (Mitchell et al. 2008), non-small-cell lung carcinoma (Chen et al. 2008), colorectal cancer (Chen et al. 2008), type 2 diabetes (Chen et al. 2008), ovarian cancer (Resnick et al. 2009), and drug-induced liver injury (Wang et al. 2009). In a previous study, we demonstrated differential miRNA expression profiles in the plasma of POF patients (Table 1) and normal cycling women by miRNA microarray analysis. The ten upregulated miRNAs were mir-202, mir-146a, mir-125b-2*, mir-139-3p, mir-654-5p, mir-27a, mir-765, mir-23a, mir$342-3 p$, and mir-126 and the two downregulated
Table 1 General characteristics of premature ovarian failure (POF) patients and normal cycling women.

\begin{tabular}{llr}
\hline & \multicolumn{1}{c}{ POF } & \multicolumn{1}{c}{ Normal } \\
\hline Age & $30.33 \pm 4.33$ & $29.80 \pm 5.82$ \\
BMl $\left(\mathrm{kg} / \mathrm{m}^{2}\right)$ & $21.74 \pm 1.43$ & $20.76 \pm 1.68$ \\
FSH $(\mathrm{mlU} / \mathrm{ml})$ & $69.48 \pm 22.88$ & $5.73 \pm 1.24$ \\
\hline
\end{tabular}

miRNAs were let-7c and mir-144 (Zhou et al. 2011). Nevertheless, the signaling pathways that regulate the expression of miRNAs during POF and the function of individual miRNAs in granulosa cell apoptosis remain unknown.

$X$-linked inhibitor of apoptosis protein (XIAP) exerts an antiapoptotic function through the direct inhibition of caspase- 3 and modulating the mitochondrial death pathway by binding Smac/DIABLO (Asselin et al. 2001, Siegel et al. 2011). miRNAs, such as mir-23a, can target XIAP and regulate its function (Siegel et al. 2011). XIAP promotes the development of rat granulosa cells and ovarian follicles via its antiapoptotic function (Andreu et al. 1998, Li et al. 1998). However, the relationship between mir-23a and XIAP in granulosa cells during follicular development and atresia remains unclear.

In this study, we performed a bioinformatic analysis of miRNA-regulated signaling pathways and related genes on the basis of miRNA expression profiles. Additionally, the role of the differentially expressed mir-23a in granulosa cell apoptosis was also explored. Herein, we demonstrate that mir-23a, which we previously demonstrated to be differentially expressed in plasma of women with POF, induces apoptosis in cultured human granulosa cells. Furthermore, we provide evidence that this occurs via downregulation of XIAP at both the mRNA and protein levels, with a subsequent increase in caspase-3 cleavage. Taken together, our findings suggest a novel mechanism through which mir-23a affects granulosa cell apoptosis, which may help to explain the potential role of mir-23a in the pathogenesis of POF.

\section{Results}

\section{Gene Ontology category}

In our previous study, the differential miRNA profile in the plasma of POF patients and normal controls has been studied using miRNA microarray analysis (Zhou et al. 2011). Briefly, total RNA from the plasma of three POF patients was compared with three normal specimens using a customized miRNA microarray, which contained 821 human miRNAs from the miRNA Registry. Primary miRNA expression profiling with microarray identified 29 miRNAs based on the $P$ value, 12 of which were differentially expressed between POF patients and normal women. The 12 upregulated miRNAs were 
mir-202, mir-146a, mir-125b-2*, mir-139-3p, mir-654$5 p$, mir-27a, mir-765, mir-23a, mir-342-3p, and mir-126, and the two downregulated miRNAs were let-7c and mir-144. For validation of these findings, miRNAs were quantified using qRT-PCR analysis in the plasma of 39 POF patients and 20 normal women. Consistent with the microarray data, mir-146a, mir-27a, mir-23a, and mir126 were highly expressed in the plasma from POF patients compared with the controls, with a fold change of 5.19, 2.98, 2.75, and 2.29 respectively.

The differentially expressed miRNAs were classified into different functional categories according to Gene Ontology (GO) analysis of biological process. The top six GO categories for upregulated genes were i) AKT signaling pathway, ii) regulation of mitochondrial membrane permeability, iii) steroid hormone receptor signaling pathway, iv) activation of mitogen-activated protein kinase kinase (MAPKK) activity, v) positive regulation of $N F-\kappa B$ transcription factor activity, and vi) induction of apoptosis by extracellular signals and apoptosis (Fig. 1A). The six primary GO categories for downregulated genes were i) AKT signaling pathway, ii) regulation of $\mathrm{Wnt}$ receptor signaling pathway, iii) regulation of growth, estrogen receptor signaling pathway, iv) induction of apoptosis by intracellular signals, v) androgen receptor signaling pathway, and vi) regulation of assembly reaction factor protein signal transduction (Fig. 1B).

\section{Pathway analysis and the miRNA-mRNA regulatory networks}

The Kyoto Encyclopedia of Genes and Genomes (KEGG) pathway analysis for upregulated genes showed that the genes were largely involved in five pathways: i) ErbB signaling pathway, ii) p53 signaling pathway, iii) MAPK signaling pathway, iv) transforming growth factor $\beta$ (TGF $\beta$ ) signaling pathway, and $v$ ) apoptosis (Fig. 2A). The KEGG pathway analysis for downregulated genes showed that the genes were more related to the mammalian target of the following five pathways: i) rapamycin (mTOR) signaling pathway, ii) TGF $\beta$ signaling pathway, iii) MAPK signaling pathway, iv) p53 signaling pathway, and (v) vascular endothelial growth factor signaling pathway (Fig. 2B).

The miRNA-mRNA regulatory networks are shown in Fig. 3, which distinguished the putative target mRNAs between upregulated and downregulated miRNAs. Three overexpressed miRNAs (mir-27a, mir-23a, and mir-202) showed 44, 33, and 28 target mRNAs respectively. Possible mir-23a-regulated genes included the antiapoptotic gene XIAP and pro-apoptotic genes phosphatase and tensin homolog deleted on chromosome 10 (PTEN), and caspase-7. These findings suggest that mir-23a may play a role in the regulation of granulosa cell apoptosis. The two downregulated miRNAs (mir-144 and let-7c) showed 27 and 41 target mRNAs, respectively, including p53, caspase-3, and PTEN.
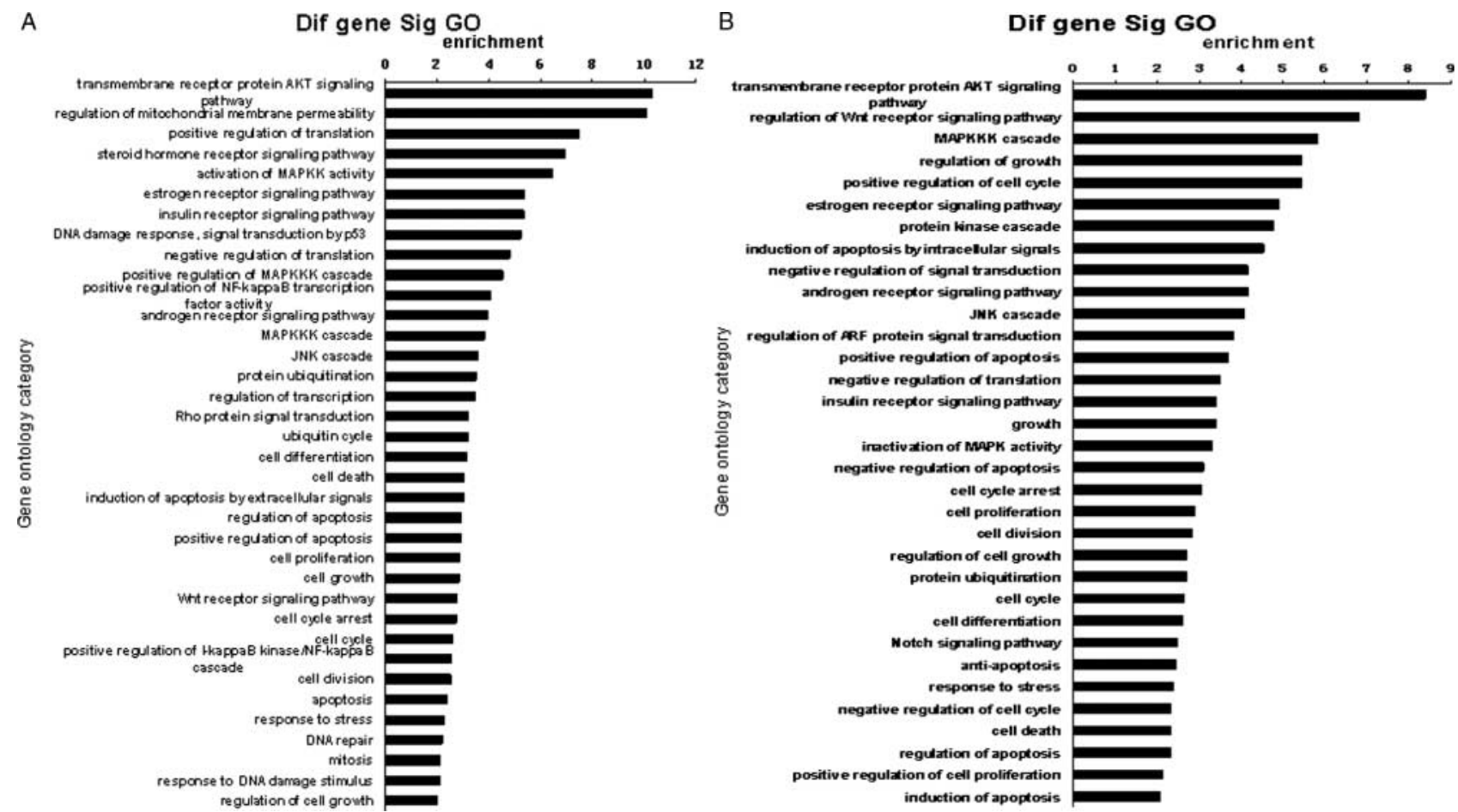

Figure 1 GOs targeted by miRNAs. (A and B) GOs targeted by the ten upregulated miRNAs and the two downregulated miRNAs respectively. All these GOs show an increased enrichment in each category by these miRNAs. The vertical axis is the GO category, and the horizontal axis is the enrichment of GO. 
A

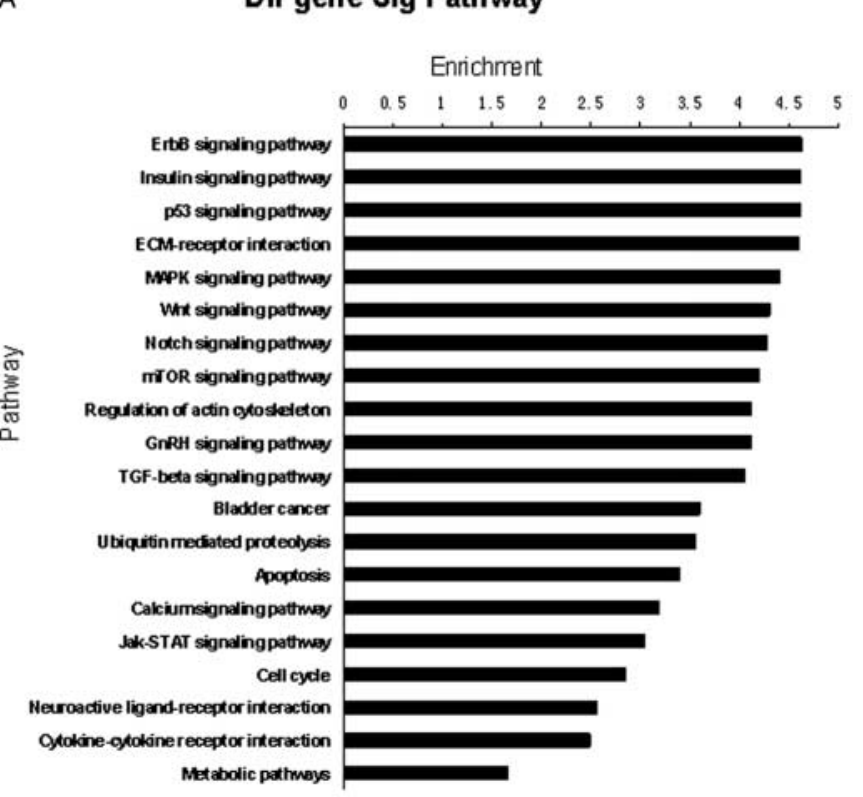

B

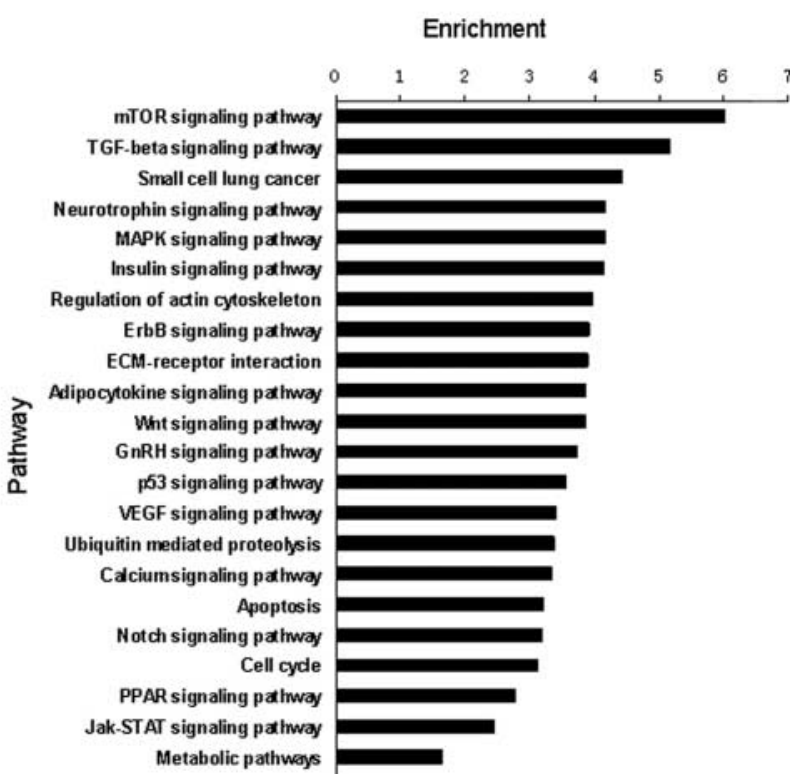

Figure 2 Pathway analysis based on miRNA-targeted genes. (A and B) Main pathways targeted by upregulated and downregulated miRNAs respectively. The vertical axis is the pathway category and the horizontal axis is the degree of enrichment of the pathway.

\section{Mir-23a-induced apoptosis in human granulosa cells}

To determine the effect of mir-23a on apoptosis in granulosa cells, the percentage of apoptotic cells in human granulosa cells transfected with pre-mir-23a or control pre-miRNA was assessed based on Hoechst 33258 staining. Mir-23a overexpression increased the rate of apoptosis in human granulosa cells. To further determine the role of mir-23a in apoptosis of granulosa cells, mir-23a inhibitor was transfected into granulosa cells. As shown in Fig. 4A, mir-23a inhibitor significantly decreased pre-mir-23a-induced apoptosis in granulosa cells $(P<0.05)$. These findings suggest that mir-23a induces apoptosis in human granulosa cells.

\section{The effects of miR-23a on XIAP and caspase-3 expression in human granulosa cells}

Recent studies have shown that mir-23a can induce caspase-dependent and -independent apoptosis in human embryonic kidney cells, both of which occur via the mitochondrial membrane disruption pathway (Chhabra et al. 2009). The KEGG pathway analysis demonstrated that mir-23a may regulate the antiapoptotic function of XIAP (Fig. 3). To investigate whether and how mir-23a is involved in granulosa cell apoptosis, the expression of XIAP and caspase-3 mRNA and protein in human granulosa cells after mir-23a overexpression was examined by RT-PCR and western blotting respectively. XIAP mRNA and protein contents were significantly downregulated in granulosa cells transfected with pre-mir-23a compared with the control pre-miRNA-transfected cells. Furthermore, caspase-3 protein content was significantly decreased, with a corresponding increase in the cleaved caspase-3 level after transfection of pre-mir-23a, suggesting caspase-3 cleavage and activation. However, caspase-3 mRNA level was not altered after transfection of pre-mir-23a, suggesting that mir-23a regulates XIAP, not caspase-3, at both transcriptional and translational levels (Fig. 4B).

To confirm that mir-23a directly and specifically regulates $X I A P$ expression, the mir-23a inhibitor or its control was transfected into granulosa cells. mir-23a inhibitor significantly increased XIAP mRNA and protein expression. In contrast, there was no change in caspase3 protein and mRNA level (Fig. 5). These findings suggest that mir-23a induces apoptosis by decreasing XIAP expression at both transcriptional and translational levels, with a subsequent cleavage of caspase-3.

\section{Discussion}

Recruitment of growing follicles, atresia, ovulation, and luteal tissue formation and regression are dynamically regulated events that regenerate on a cyclical basis in the ovary (Carletti \& Christenson 2009). These events involve dynamic changes in cellular growth, angiogenesis, steroidogenesis, cell cycle, and apoptosis and are accurately regulated at the endocrine and tissue levels (Carletti \& Christenson 2009). Defects in the regulatory networks result in ovarian failure such as POF due to disruption of folliculogenesis, blockage of ovulation, and loss of oocytes via apoptosis. Understanding the molecular events during folliculogenesis and atresia 


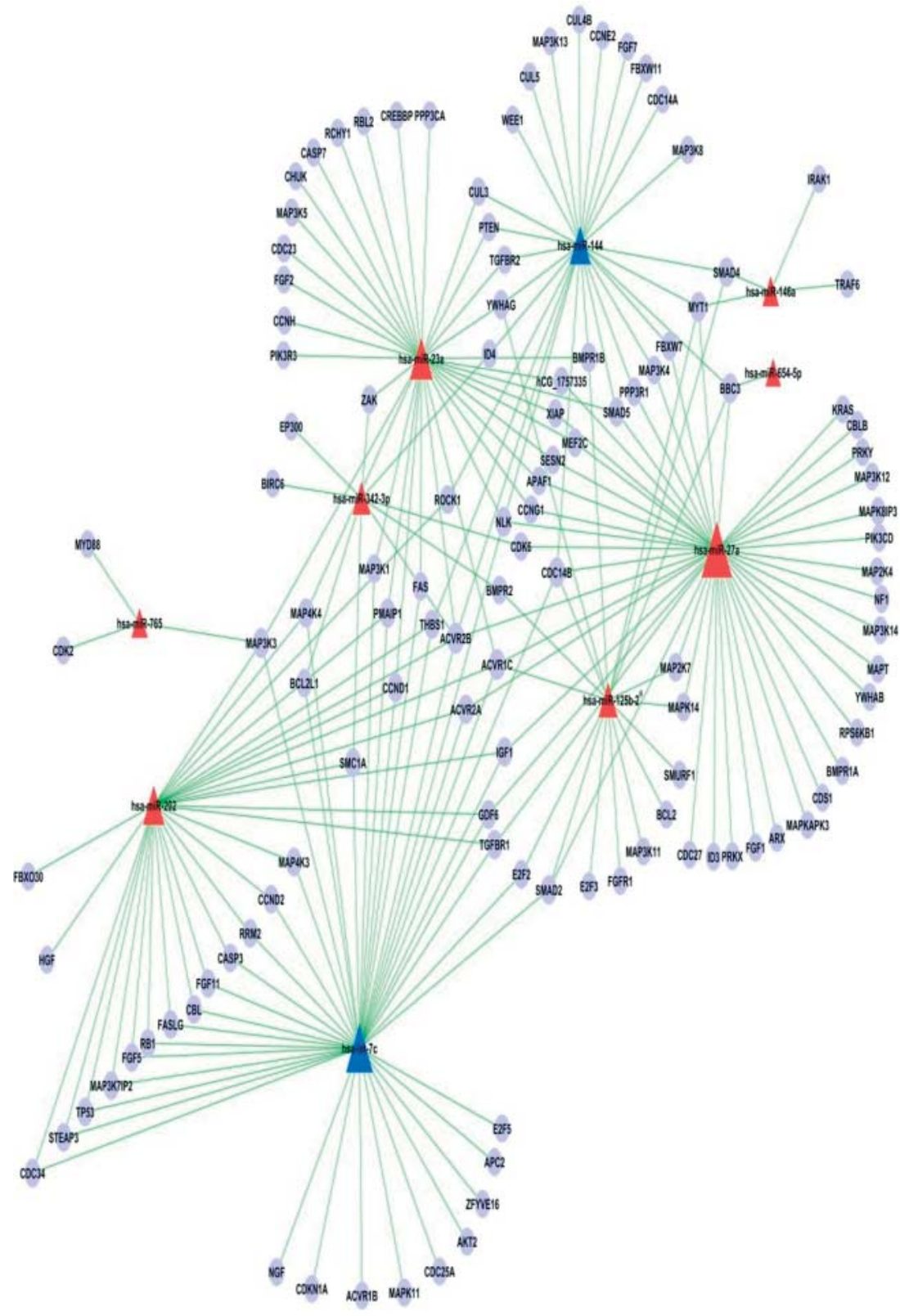

Figure 3 miRNA-mRNA network. Triangles represent miRNAs, and gray oval cycle nodes represent mRNAs. The green lines show the inhibitory effect of miRNAs on mRNAs. The red triangles represent upregulated miRNAs and blue triangles represent downregulated miRNAs. will provide insights into enhancing reproductive efficiencies and alleviating deficiencies (Carletti \& Christenson 2009).

The recent identification of miRNAs as an important posttranscriptional gene regulator has led to an explosion in our knowledge of the role of posttranscriptional gene regulation in reproductive organ such as the ovary. In addition, miRNAs have also been recognized to be involved in crucial cell processes, such as apoptosis, differentiation, and oncogenesis by regulating signal transduction pathways (Ambros 2004). In a previous study, we identified several differentially expressed miRNAs in the plasma of POF patients and normally cycling women, including ten upregulated miRNAs (mir202, mir-146a, mir-125b-2*, mir-139-3p, mir-654-5p, mir-27a, mir-765, mir-23a, mir-342-3p, and mir-126) and two downregulated miRNAs (let-7c and mir-144). We also verified four differentially expressed miRNAs (mir-146a, mir-27a, mir-23a, and mir-126) in POF and found that the results were consistent with miRNA microarray analysis (Zhou et al. 2011).

In order to further determine the function of the differentially expressed plasma miRNAs, GO analysis and KEGG pathway annotation were used to analyze their target gene pools. GO organized genes targeted by differential miRNAs into hierarchical categories based on biological processes and then outlined the roles of miRNAs. In this study, GO analysis illustrates that these miRNAs are most related to the AKT signaling pathway, regulation of mitochondrial membrane permeability, 


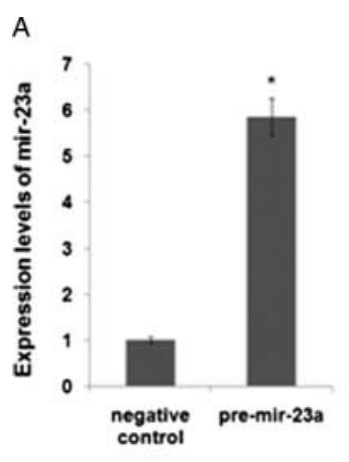

B
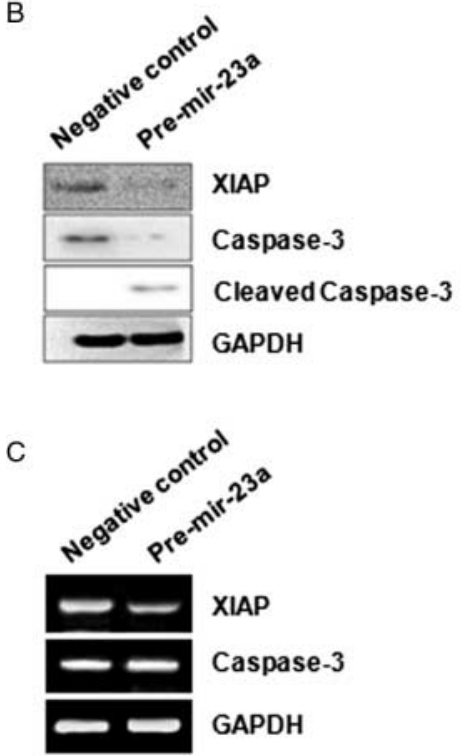
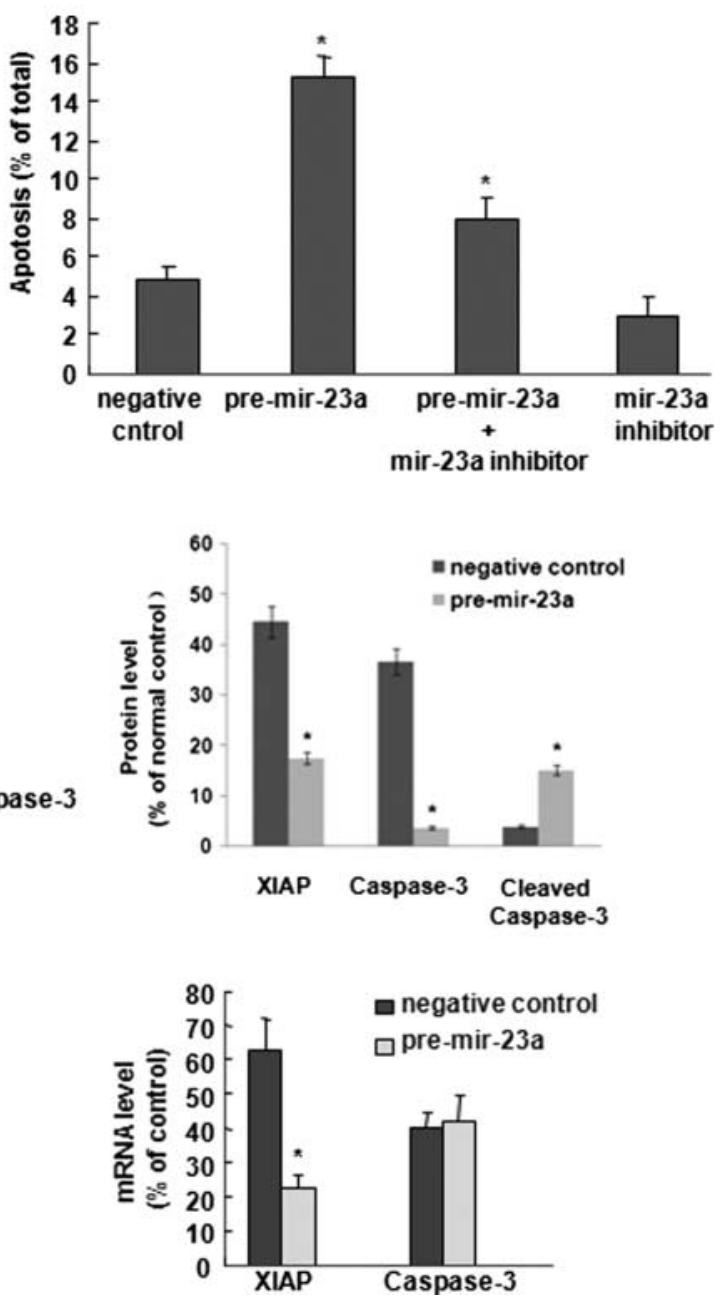

Figure 4 Mir-23a is pro-apoptotic in human granulosa cells. (A) Pre-mir-23a increased apoptosis and mir-23a inhibitor decreased apoptosis in human granulosa cells as assessed by Hoechst 33258 staining $\left({ }^{*} P<0.05\right.$, $t$-test, $\left.n=3\right)$.

(B) Pre-mir-23a decreased XIAP and caspase-3 protein content and increased cleaved caspase- 3 content in human granulosa cells $(* P<0.05$, $t$-test, $n=3)$. GAPDH was included as a loading control. (C) XIAP mRNA level was decreased $\left({ }^{*} P<0.05, t\right.$-test, $\left.n=3\right)$ and there was no change in caspase- 3 mRNA level $(P>0.05, t$-test, $n=3)$ after transfection of pre-mir-23a. steroid hormone receptor signaling pathway, activation of MAPKK activity, positive regulation of NF- $\kappa B$ transcription factor activity, induction of apoptosis by extracellular signals, apoptosis, and cell growth (Fig. 1). KEGG annotation showed that survival pathways (TGF $\beta$ and $\mathrm{mTOR}$ ), importantly proliferative (ErbB, MAPK, Wnt, and cell cycle) and apoptotic (p53 signaling pathway and apoptosis) signaling pathways, were most abundant among the significantly enriched ones (Fig. 2), which was in accordance with the GO analysis. This functional identity revealed by different bioinformatic methods suggested that miRNAs may have regulatory effects on proliferation and apoptosis of granulosa cells by affecting the signaling pathways as mentioned earlier.

The miRNA-mRNA regulatory network analysis further integrated the bioinformatic observations and then outlined the main targets of miRNAs (Fig. 3). Mir$27 \mathrm{a}$ and mir-23a have 44 and 33 target mRNAs, respectively, that exhibit more target mRNAs compared with the other miRNAs. The mir-23a 27a 24-2 cluster has been shown to play important roles in several processes during normal and pathologic states and is tightly related to the cell cycle, proliferation, differentiation, apoptosis, hematopoiesis, and cardiac hypertrophy (Huang et al. 2008, Chhabra et al. 2010). Studies have illustrated that the mir-23a 27a 24-2 cluster functions as a growth-promoting and antiapoptotic factor targeting the Smad pathway in hepatocellular carcinoma cells (Huang et al. 2008). However, a recent study demonstrated that the mir-23a $\sim 27 \mathrm{a} \sim 24-2$ cluster could induce caspase-dependent and -independent apoptosis in human embryonic kidney cells (Chhabra et al. 2009). The pro-apoptotic and antiapoptotic nature of the mir-23a $27 a \sim 24-2$ cluster suggests that this cluster may play different roles under different physiological and pathological conditions. It is of great interest to identify the function of mir-23a in granulosa cells and follicular atresia.

In this investigation, several lines of evidence suggest that mir-23a promotes apoptosis of human granulosa cells. First, overexpression of mir-23a in granulosa cells can induce downregulation of antiapoptotic XIAP and increase the level of the pro-apoptotic cleaved form of caspase-3 as measured by western blotting analysis (Fig. 4A). Secondly, the percentage of apoptotic cells is significantly increased in mir-23a-treated granulosa cells 
A

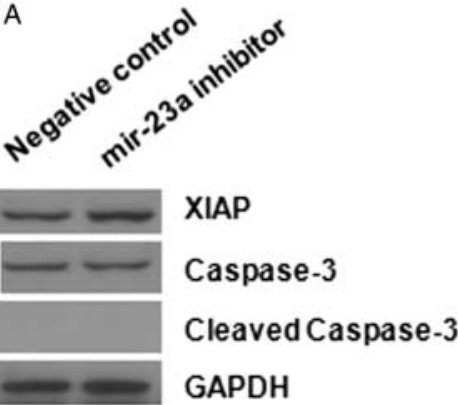

B

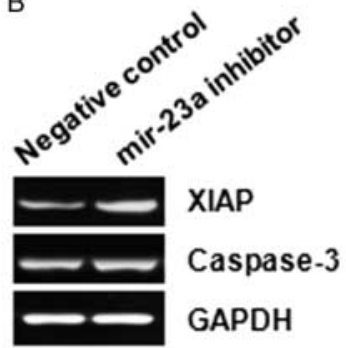

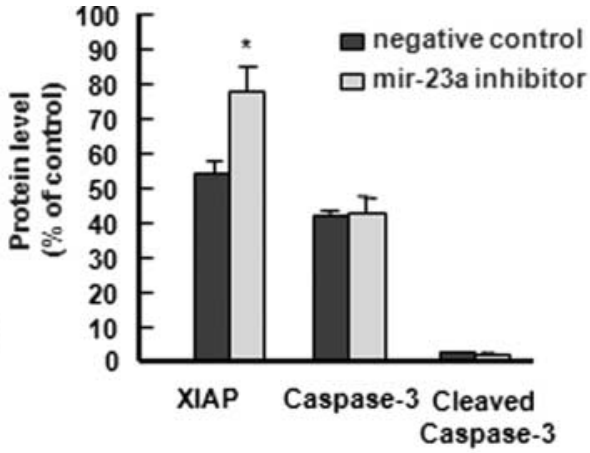

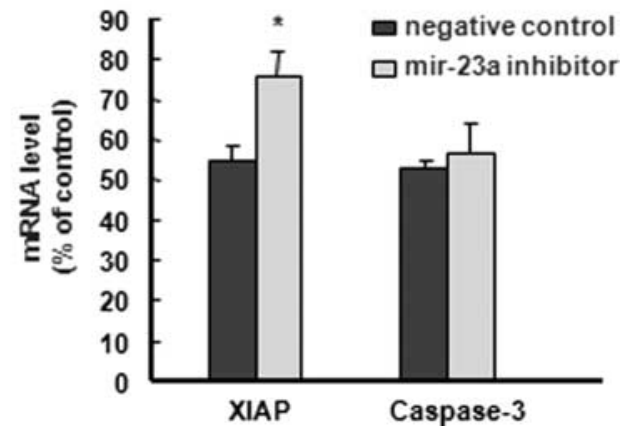

Figure 5 Mir-23a inhibitor increased XIAP protein and mRNA level. (A) XIAP protein content increased after transfection of mir-23a inhibitor, but there was no change in caspase- 3 protein content $\left({ }^{*} P<0.05, t\right.$-test, $\left.n=3\right)$. (B) $X I A P$ mRNA level increased after transfection of mir-23a inhibitor, but there was no change in caspase-3 mRNA level $(* P<0.05, t$-test, $n=3)$. compared with control miRNA-treated granulosa cells based on Hoechst 33258 staining (Fig. 4D). Furthermore, mir-23a inhibitor blocked mir-23a-induced apoptosis and decreased XIAP mRNA expression, with no effect on caspase-3 mRNA expression. XIAP is the endogenous inhibitor of caspase-3, and decreasing XIAP expression by mir-23a may contribute to enhancing caspase- 3 activity (Siegel et al. 2011). These results provide clear evidence, for the first time, that mir-23a promotes apoptosis of granulosa cells via decreasing XIAP expression, which may contribute to the etiology of POF.

In conclusion, some plasma miRNAs are differentially expressed in POF patients and normal cycling women. Mir-23a, which is significantly upregulated in the plasma of POF patients, is essential for apoptosis induction in human granulosa cells by targeting XIAP and the caspase signaling cascade. In a word, these findings highlight the important roles of miRNAs in the nosogenesis of POF.

\section{Materials and Methods}

\section{Study participants}

All the samples were obtained following signed informed consent. All procedures for sample collection were approved by the Human Ethics Committees of Beijing Obstetrics and Gynecology Hospital, Capital Medical University, and Institute of Zoology, Chinese Academy of Sciences.

Thirty-six infertile patients, $<40$ years of age who underwent their first IVF or ICSI-embryo transfer (ET) cycle, were included in this study (Table 2). The etiology of infertility was either due to tubal or male factors. The basal FSH, $\mathrm{LH}$, estradiol $\left(\mathrm{E}_{2}\right)$, progesterone, $\mathrm{PRL}$, and testosterone levels were measured on day 3 of the cycle before their stimulation cycle. The long protocol of GNRH-a downregulation in the midluteal phase was used for the patients undergoing IVF or ICSI-ET. Recombinant FSH (150-225 IU) was administered on day 3 of the cycle. HCG (10000 IU) was given when the leading follicle was $\geq 18 \mathrm{~mm}$ in diameter and there were at least two follicles $\geq 16 \mathrm{~mm}$ in diameter. Oocyte retrieval was arranged after $36 \mathrm{~h}$. Samples of follicular fluid, for the isolation of ovarian granulosa cells, were collected from these patients undergoing stimulation cycles of IVF or ICSI-ET at the Department of Human Reproductive Medicine, Beijing Obstetrics and Gynecology Hospital.

\section{Isolation of human granulosa cells}

Isolation of human ovarian granulosa cells from follicular fluid was performed as described by Gillott et al. (2008). Briefly, all granulosa cells were disaggregated by incubating with $10 \%$ hyaluronidase for $15 \mathrm{~min}$ at $37^{\circ} \mathrm{C}$ and separated from the red blood cells and lymphocytes by density gradient centrifugation over 50\% Percoll (Sigma) for $15 \mathrm{~min}$ at $1000 \mathrm{~g}$. The granulosa cells at the interface were harvested. After being centrifuged at $500 \mathrm{~g}$ for $5 \mathrm{~min}$, cells were cultured in six-well plates $\left(\sim 10^{6}\right.$ cells/well) using Roswell Park Memorial Institute (RPMI)-1640 with glutamine and $\mathrm{NaHCO}_{3}$ supplemented with $10 \%$ fetal calf plasma and $1 \%$ antibiotic-antimycotic (penicillin and streptomycin; Sigma) at $37^{\circ} \mathrm{C}$ with $5 \% \mathrm{CO}_{2}$. Cultures were maintained for $18 \mathrm{~h}$ until the culture media were changed and any nonadherent cells were removed.

\section{Transfection with miRNAs and miRNA inhibitor}

Ovarian granulosa cells were transfected with $60 \mathrm{pM}$ pre-mir23a or pre-mir-negative control (Ambion Inc., Austin, TX, USA), as well as mir-23a inhibitor or its control (Exiqon, Vedbaek, Denmark), in six-well plates according to the 
Table 2 General characteristics of the study population.

\begin{tabular}{lc}
\hline Age (years) & $31.83 \pm 3.63$ \\
Menstrual cycle length (days) & $31.61 \pm 3.73$ \\
Duration of infertility (years) & $4.17 \pm 2.50$ \\
Antral follicle number & $11.11 \pm 2.89$ \\
$\mathrm{BMI}\left(\mathrm{kg} / \mathrm{m}^{2}\right)$ & $21.37 \pm 1.73$ \\
$\mathrm{Basal} \mathrm{FSH}(\mathrm{mlU} / \mathrm{ml})$ & $5.89 \pm 1.03$ \\
Basal LH $(\mathrm{mlU} / \mathrm{ml})$ & $4.77 \pm 1.96$ \\
$\mathrm{FSH} / \mathrm{LH}$ & $1.47 \pm 0.73$ \\
Basal E $(\mathrm{pg} / \mathrm{ml})$ & $26.41 \pm 5.46$ \\
Basal progesterone $(\mathrm{ng} / \mathrm{ml})$ & $0.83 \pm 0.18$ \\
Basal PRL $(\mathrm{ng} / \mathrm{ml})$ & $14.16 \pm 7.08$ \\
Basal testosterone $(\mathrm{ng} / \mathrm{dl})$ & $39.43 \pm 8.50$ \\
Peak $\mathrm{E}_{2}$ & $2776.949 \pm 1156.806$ \\
Peak LH & $2.44 \pm 1.56$ \\
Peak P & $1.62 \pm 0.75$ \\
Retrieved oocytes $(n)$ & $10.83 \pm 2.31$ \\
Metaphase Il oocytes $(n)$ & $9.83 \pm 1.82$ \\
\hline
\end{tabular}

manufacturer's instructions. Transfection was performed using Lipofectamine 2000 (Invitrogen, Carlsbad, CA, USA) when the granulosa cells in six-well plates reached $50-60 \%$ confluence. Cells were incubated with Opti-MEM (Invitrogen) for $6 \mathrm{~h}$ and then with fresh RPMI-1640 medium containing 10\% fetal bovine serum for $24 \mathrm{~h}$. Total RNAs and proteins were prepared $48 \mathrm{~h}$ after transfection and used for quantitative real-time-PCR or western blot analysis.

\section{Western blotting analysis}

Western blotting was done as described by Yang et al. (2006). Briefly, total protein extracts were prepared using whole-cell lysis buffer $(50 \mathrm{mM}$ HEPES, $150 \mathrm{mM} \mathrm{NaCl}, 1 \mathrm{mM}$ EGTA, $10 \mathrm{mM}$ sodium pyrophosphate, $1.5 \mathrm{mM} \mathrm{MgCl}, 100 \mathrm{mM}$ sodium fluoride, $10 \%$ glycerol, and $1 \%$ Triton X-100) containing an inhibitor mixture $(1 \mathrm{mM}$ phenylmethylsulfonyl fluoride, $10 \mu \mathrm{g} / \mathrm{ml}$ aprotinin, and $1 \mathrm{mM}$ sodium orthovanadate). Protein concentrations were determined using a standard Bradford assay, and $50 \mu \mathrm{g}$ total protein were subjected to SDSPAGE followed by electrotransfer onto nitrocellulose membranes. Membranes were incubated overnight at $4{ }^{\circ} \mathrm{C}$ with primary antibodies against human XIAP (R\&D, Minneapolis, MN, USA), human caspase-3 (Santa Cruz Biotechnology, Inc, Santa Cruz, CA, USA), or human glyceraldehyde-3-phosphate dehydrogenase (GAPDH; Abcam) followed by incubation with secondary antibodies. Signals were developed using the enhanced chemiluminescence system (Pierce, Rockford, IL, USA).

\section{Quantitative real time-PCR}

Real-time PCR was performed using a standard SYBR Premix Ex Taq kit (Takara Bio Inc., Shiga, Japan) on an Applied Biosystems 7500 fast real-time PCR System (Applied Biosystems, Carlsbad, $\mathrm{CA}, \mathrm{USA})$. The $10 \mu \mathrm{l}$ PCR reaction system included $5 \mu \mathrm{l}$ SYBR Green PCR Master Mix, $1 \mu \mathrm{l}$ cDNA, $0.2 \mu \mathrm{l}$ ROX Reference Dye II, $0.5 \mu$ l specific primer (Table 1), and $3.3 \mu$ R Rase-free water. The reactions were performed in a 96 -well plate at $95{ }^{\circ} \mathrm{C}$ for $30 \mathrm{~s}$; followed by 45 cycles at $95^{\circ} \mathrm{C}$ for $5 \mathrm{~s}, 60^{\circ} \mathrm{C}$ for $10 \mathrm{~s}$, and $72{ }^{\circ} \mathrm{C}$ for $25 \mathrm{~s}$; and finally $95^{\circ} \mathrm{C}$ for $15 \mathrm{~s}, 60{ }^{\circ} \mathrm{C}$ for $1 \mathrm{~min}$, and $95^{\circ} \mathrm{C}$ for $15 \mathrm{~s}$. All reactions were run in triplicate. The threshold cycle was defined as the fractional cycle number at which the fluorescence passed the fixed threshold. Negative control reactions without RT reaction and template were also performed.

\section{RNA isolation and RT-PCR}

Total RNA was isolated from human ovarian granulosa cells using TRIzol reagent according to the manufacturer's instructions. RNA $(2 \mu \mathrm{g})$ was used for cDNA synthesis by RT as previously reported (Yang et al. 2004). The cDNAs obtained were amplified using specific primers (Asselin et al. 2001, Chen et al. 2009) as follows: XIAP (389 bp), 5'-GAA GAC CCT TGG GAA CAA CA-3' (sense) and 5'-CGC CTT AGC TGC TCT TCA GT-3' (antisense); caspase-3 (445 bp), forward, 5'-CACAATAGCACCCATCCG-3' (sense) and $5^{\prime}$-GGGACATCAGTCGCTTCA-3' (antisense); and GADPH (230 bp), 5'-ACG GAT TTG GTC GTA TTG GG-3' (sense) and 5'-TGA TTT TGG AGG GAT CTC GC-3' (antisense).

To detect the mRNA of XIAP and caspase-3, PCR was performed at $94{ }^{\circ} \mathrm{C}$ for $15 \mathrm{~s}, 51^{\circ} \mathrm{C}$ for $30 \mathrm{~s}$, and $68{ }^{\circ} \mathrm{C}$ for $90 \mathrm{~s}$ for 30 cycles. GAPDH expression was used as an internal control.

\section{Assessment of apoptosis}

Cells were harvested and stained using Hoechst 33258 as previously reported (Wang et al. 2006). At least 200 cells in several selected areas were counted in each treatment group. Cells were counted with the counter 'blinded' to the sample identity to avoid experimental bias. In contrast to normal cells, the nuclei of apoptotic cells have highly condensed chromatin that is stained by Hoechst 33258 . This can take the form of crescents around the perimeter of the nucleus, or the entire nucleus can appear to be one or a group of featureless, bright spherical beads. These morphological changes in the nuclei of apoptotic cells can be visualized by fluorescence microscopy.

\section{GO analysis}

GO analysis was applied to analyze the main function of the differentially expressed genes according to the GO, which is the key functional classification of NCBI (Ashburner et al. 2000). Generally, Fisher's exact test and $\chi^{2}$-test were used to classify the GO category. The false discovery rate (FDR; Dupuy et al. 2007) was calculated to correct the $P$ value. The smaller the FDR, the smaller the error would be in judging the $P$ value. The FDR was defined as FDR $=1-N_{\mathrm{k}} / T$, where $N_{\mathrm{k}}$ refers to the number of Fisher's test $P$ values less than $\chi^{2}$-test $P$ values and $T$ refers to permutation test. $P$ values were computed for the GOs of all differential genes. Enrichment provides a measure of the significance of the function: as the enrichment increases, the corresponding function is more specific, which helps to indicate GOs with more concrete function description in the experiment. Within the significant category, the enrichment Re was given by:

$$
\operatorname{Re}=\frac{\left(n_{\mathrm{f}} / n\right)}{\left(N_{\mathrm{f}} / N\right)}
$$


with $n_{\mathrm{f}}$ being the number of differential genes within the particular category, $n$ being the total number of genes within the same category, $N_{f}$ being the number of differential genes in the entire microarray, and $N$ being the total number of genes in the microarray.

\section{MicroRNA-Gene Network}

The relationship of the miRNA and genes were determined by their differential expression values, and according to the interactions of miRNA and genes in the Sanger miRNA database, the MicroRNA-Gene Network was built (Joung et al. 2007). In the MicroRNA-Gene Network, the circle represents gene and the shape of square represents miRNA, and their relationship was represented by one edge. The center of the network was represented by degree. Degree means the contribution of one miRNA to the genes around or the contribution of one gene to the miRNAs around. The key miRNA and the gene in the network always have the biggest degrees (Joung et al. 2007, Shalgi et al. 2007).

\section{Pathway analysis}

Pathway analysis was used to determine the significant pathways of the differentially expressed genes according to the KEGG and Biocarta and Reatome. Still, the Fisher's exact test and $\chi^{2}$-test were used to select the significant pathways, and the threshold of significance was defined by $P$ value and FDR. The enrichment Re was calculated as in the equation mentioned earlier (Kanehisa et al. 2004, Yi et al. 2006, Draghici et al. 2007).

\section{Statistical analysis}

Results are presented as mean \pm s.E.M. of at least three independent experiments. Quantitative data were compared with a student $t$-test between the two groups. $P<0.05$ was considered statistically significant.

\section{Declaration of interest}

The authors declare that there is no conflict of interest that could be perceived as prejudicing the impartiality of the research reported.

\section{Funding}

This work was supported by funding from the Natural Science Foundation of China (grant no. 30872746) and Natural Science Foundation of Beijing (grant no. 7082034) to X Yang and a grant from Natural Science Foundation of China (grant no. 30971088) to H Wang.

\section{Acknowledgements}

The authors thank Ms Yingzhe Zhu for plasma sample collection. They also thank Drs Michael Fraser and Yifang Wang for their helpful discussion and critical reading of the manuscript. Genminix Informatics provided them technical assistance.

\section{References}

Ambros V 2004 The functions of animal microRNAs. Nature 431 350-355. (doi:10.1038/nature02871)

Andreu C, Parborell F, Vanzulli S, Chemes H \& Tesone M 1998 Regulation of follicular luteinization by a gonadotropin-releasing hormone agonist: relationship between steroidogenesis and apoptosis. Molecular Reproduction and Development 51 287-294. (doi:10.1002/(SICl)1098-2795 (199811)51:3<287::AID-MRD8 > 3.0.CO;2-L)

Ashburner M, Ball CA, Blake JA, Botstein D, Butler H, Cherry JM, Davis AP, Dolinski K, Dwight SS, Eppig JT et al. 2000 Gene ontology: tool for the unification of biology. The Gene Ontology Consortium. Nature Genetics 25 25-29. (doi:10.1038/75556)

Asselin E, Mills GB \& Tsang BK 2001 XIAP regulates Akt activity and caspase-3-dependent cleavage during cisplatin-induced apoptosis in human ovarian epithelial cancer cells. Cancer Research 61 1862-1868.

Bartel DP 2004 MicroRNAs: genomics, biogenesis, mechanism, and function. Cell 116 281-297. (doi:10.1016/S0092-8674(04)00045-5)

Beck-Peccoz P \& Persani L 2006 Premature ovarian failure. Orphanet Journal of Rare Diseases 1 9. (doi:10.1186/1750-1172-1-9)

Bernstein E, Caudy AA, Hammond SM \& Hannon GJ 2001 Role for a bidentate ribonuclease in the initiation step of RNA interference. Nature 409 363-366. (doi:10.1038/35053110)

Bernstein E, Kim SY, Carmell MA, Murchison EP, Alcorn H, Li MZ, Mills AA, Elledge SJ, Anderson KV \& Hannon GJ 2003 Dicer is essential for mouse development. Nature Genetics 35 215-217. (doi:10.1038/ng1253)

Billig H, Furuta I \& Hsueh AJ 1993 Estrogens inhibit and androgens enhance ovarian granulosa cell apoptosis. Endocrinology 133 2204-2212. (doi:10.1210/en.133.5.2204)

Billig H, Furuta I \& Hsueh AJ 1994 Gonadotropin-releasing hormone directly induces apoptotic cell death in the rat ovary: biochemical and in situ detection of deoxyribonucleic acid fragmentation in granulosa cells. Endocrinology 134 245-252. (doi:10.1210/en.134.1.245)

Carletti MZ \& Christenson LK 2009 MicroRNA in the ovary and female reproductive tract. Journal of Animal Science 87 E29-E38. (doi:10.2527/ jas.2008-1331)

Chen X, Ba Y, Ma L, Cai X, Yin Y, Wang K, Guo J, Zhang Y, Chen J \& Guo X 2008 Characterization of microRNAs in serum: a novel class of biomarkers for diagnosis of cancer and other diseases. Cell Research 18 997-1006. (doi:10.1038/cr.2008.282)

Chen BA, Lai BB, Cheng J, Xia GH, Gao F, Xu WL, Ding JH, Gao C, Sun XC, Xu CR et al. 2009 Daunorubicin-loaded magnetic nanoparticles of $\mathrm{Fe}_{3} \mathrm{O}_{4}$ overcome multidrug resistance and induce apoptosis of K562-n/ VCR cells in vivo. International Journal of Nanomedicine 4 201-208. (doi:10.2147/IJN.S7287)

Chhabra R, Adlakha YK, Hariharan M, Scaria V \& Saini N 2009 Upregulation of miR-23a $\sim 27 \mathrm{a} \sim 24-2$ cluster induces caspase-dependent and -independent apoptosis in human embryonic kidney cells. PLOS ONE4 e5848. (doi:10.1371/journal.pone.0005848)

Chhabra R, Dubey R \& Saini N 2010 Cooperative and individualistic functions of the microRNAs in the miR-23a $27 a \sim 24-2$ cluster and its implication in human diseases. Molecular Cancer 9 232. (doi:10.1186/ 1476-4598-9-232)

Chim SS, Shing TK, Hung EC, Leung TY, Lau TK, Chiu RW \& Lo YM 2008 Detection and characterization of placental microRNAs in maternal plasma. Clinical Chemistry 54 482-490. (doi:10.1373/clinchem.2007. 097972)

Choi Y, Qin Y, Berger MF, Ballow DJ, Bulyk ML \& Rajkovic A 2007 Microarray analyses of newborn mouse ovaries lacking Nobox. Biology of Reproduction 77 312-319. (doi:10.1095/biolreprod.107.060459)

Conway GS 1996 Clinical manifestations of genetic defects affecting gonadotrophins and their receptors. Clinical Endocrinology 45 657-663. (doi:10.1046/j.1365-2265.1996.8680879.x)

Draghici S, Khatri P, Tarca AL, Amin K, Done A, Voichita C, Georgescu C \& Romero R 2007 A systems biology approach for pathway level analysis. Genome Research 17 1537-1545. (doi:10.1101/gr.6202607)

Dupuy D, Bertin N, Hidalgo CA, Venkatesan K, Tu D, Lee D, Rosenberg J, Svrzikapa N, Blanc A, Carnec A et al. 2007 Genome-scale analysis of in vivo spatiotemporal promoter activity in Caenorhabditis elegans. Nature Biotechnology 25 663-668. (doi:10.1038/nbt1305) 
Fiedler SD, Carletti MZ, Hong X \& Christenson LK 2008 Hormonal regulation of microRNA expression in periovulatory mouse mural granulosa cells. Biology of Reproduction 79 1030-1037. (doi:10.1095/ biolreprod.108.069690)

Gilad S, Meiri E, Yogev Y, Benjamin S, Lebanony D, Yerushalmi N, Benjamin H, Kushnir M, Cholakh H, Melamed N et al. 2008 Serum microRNAs are promising novel biomarkers. PLOS ONE 3 e3148. (doi:10.1371/journal.pone.0003148)

Gillott DJ, Eldib A, lammarrone E, Leung KY, Thornhill AR \& Grudzinskas JG 2008 Glycolytic enzyme expression in human granulosa cells. Fertility and Sterility 90 1405-1410. (doi:10.1016/j.fertnstert.2007. 08.053)

Hong X, Luense LJ, McGinnis LK, Nothnick WB \& Christenson LK 2008 Dicer1 is essential for female fertility and normal development of the female reproductive system. Endocrinology 149 6207-6212. (doi:10.1210/en.2008-0294)

Huang S, He X, Ding J, Liang L, Zhao Y, Zhang Z, Yao X, Pan Z, Zhang P, Li J et al. 2008 Upregulation of miR-23a approximately 27 a approximately 24 decreases transforming growth factor-beta-induced tumor-suppressive activities in human hepatocellular carcinoma cells. International Journal of Cancer 123 972-978. (doi:10.1002/ijc.23580)

Joung JG, Hwang KB, Nam JW, Kim SJ \& Zhang BT 2007 Discovery of microRNA-mRNA modules via population-based probabilistic learning. Bioinformatics 23 1141-1147. (doi:10.1093/bioinformatics/btm045)

Kanehisa M, Goto S, Kawashima S, Okuno Y \& Hattori M 2004 The KEGG resource for deciphering the genome. Nucleic Acids Research 32 D277-D280. (doi:10.1093/nar/gkh063)

van Kasteren YM, Hundscheid RD, Smits AP, Cremers FP, van Zonneveld P \& Braat DD 1999 Familial idiopathic premature ovarian failure: an overrated and underestimated genetic disease? Human Reproduction 14 2455-2459. (doi:10.1093/humrep/14.10.2455)

Kim HJ, Cui XS, Kim EJ, Kim WJ \& Kim NH 2006 New porcine microRNA genes found by homology search. Genome 49 1283-1286. (doi:10.1139/ g06-120)

Li J, Kim JM, Liston P, Li M, Miyazaki T, Mackenzie AE, Korneluk RG \& Tsang BK 1998 Expression of inhibitor of apoptosis proteins (IAPs) in rat granulosa cells during ovarian follicular development and atresia. Endocrinology 139 1321-1328. (doi:10.1210/en.139.3.1321)

Mitchell PS, Parkin RK, Kroh EM, Fritz BR, Wyman SK, PogosovaAgadjanyan EL, Peterson A, Noteboom J, O'Briant KC, Allen A et al. 2008 Circulating microRNAs as stable blood-based markers for cancer detection. PNAS 105 10513-10518. (doi:10.1073/pnas.0804549105)

Murchison EP, Stein P, Xuan Z, Pan H, Zhang MQ, Schultz RM \& Hannon G 2007 Critical roles for Dicer in the female germline. Genes and Development 21 682-693. (doi:10.1101/gad.1521307)

Otsuka M, Zheng M, Hayashi M, Lee JD, Yoshino O, Lin S \& Han J 2008 Impaired microRNA processing causes corpus luteum insufficiency and infertility in mice. Journal of Clinical Investigation 118 1944-1954. (doi:10.1172/JCl33680)

Paroo Z, Liu Q \& Wang X 2007 Biochemical mechanisms of the RNAinduced silencing complex. Cell Research 17 187-194.

Resnick KE, Alder H, Hagan JP, Richardson DL, Croce CM \& Cohn DE 2009 The detection of differentially expressed microRNAs from the serum of ovarian cancer patients using a novel real-time PCR platform. Gynecologic Oncology 112 55-59. (doi:10.1016/j.ygyno.2008.08.036)

Ro S, Song R, Park C, Zheng H, Sanders KM \& Yan W 2007 Cloning and expression profiling of small RNAs expressed in the mouse ovary. $R N A$ 13 2366-2380. (doi:10.1261/rna.754207)

Shalgi R, Lieber D, Oren M \& Pilpel Y 2007 Global and local architecture of the mammalian microRNA-transcription factor regulatory network. PLoS Computational Biology 3 e131. (doi:10.1371/journal.pcbi.0030131)
Siegel C, Li J, Liu F, Benashski SE \& McCullough LD 2011 miR-23a regulation of X-linked inhibitor of apoptosis (XIAP) contributes to sex differences in the response to cerebral ischemia. PNAS 108 11662-11667. (doi:10.1073/pnas.1102635108)

Snowdon DA, Kane RL, Beeson WL, Burke GL, Sprafka JM, Potter J, Iso H, Jacobs DR Jr \& Phillips RL 1989 Is early natural menopause a biologic marker of health and aging? American Journal of Public Health $\mathbf{7 9}$ 709-714. (doi:10.2105/AJPH.79.6.709)

Tang F, Kaneda M, O'Carroll D, Hajkova P, Barton SC, Sun YA, Lee C, Tarakhovsky A, Lao K \& Surani MA 2007 Maternal microRNAs are essential for mouse zygotic development. Genes and Development 21 644-648. (doi:10.1101/gad.418707)

Tsujiura M, Ichikawa D, Komatsu S, Shiozaki A, Takeshita H, Kosuga T, Konishi H, Morimura R, Deguchi K, Fujiwara H et al. 2010 Circulating microRNAs in plasma of patients with gastric cancers. British Journal of Cancer 102 1174-1179. (doi:10.1038/sj.bjc.6605608)

Vegetti W, Marozzi A, Manfredini E, Testa G, Alagna F, Nicolosi A, Caliaria I, Taborellid M, Tibilettid MG, Dalpráb L et al. 2000 Premature ovarian failure. Molecular and Cellular Endocrinology 161 53-57. (doi:10.1016/S0303-7207(99)00224-5)

Wang H, Jiang JY, Zhu C, Peng C \& Tsang BK 2006 Role and regulation of nodal/activin receptor-like kinase 7 signaling pathway in the control of ovarian follicular atresia. Molecular Endocrinology 20 2469-2482. (doi:10.1210/me.2005-0446)

Wang K, Zhang S, Marzolf B, Troisch P, Brightman A, Hu Z, Hood LE \& Galas DJ 2009 Circulating microRNAs, potential biomarkers for druginduced liver injury. PNAS 106 4402-4407. (doi:10.1073/pnas. 0813371106)

Yamamoto $\mathrm{Y}$, Kosaka N, Tanaka M, Koizumi F, Kanai $\mathrm{Y}$, Mizutani T, Murakami Y, Kuroda M, Miyajima A, Kato T et al. 2009 MicroRNA-500 as a potential diagnostic marker for hepatocellular carcinoma. Biomarkers 14 529-538. (doi:10.3109/13547500903150771)

Yang X, Zheng F, Xing H, Gao Q, Wei W, Lu Y, Wang S, Zhou J, Hu W \& Ma D 2004 Resistance to chemotherapy-induced apoptosis via decreased caspase-3 activity and overexpression of antiapoptotic proteins in ovarian cancer. Journal of Cancer Research and Clinical Oncology 130 423-428. (doi:10.1007/s00432-004-0556-9)

Yang X, Fraser M, Moll UM, Basak A \& Tsang BK 2006 Akt-mediated cisplatin resistance in ovarian cancer: modulation of p53 action on caspase-dependent mitochondrial death pathway. Cancer Research $\mathbf{6 6}$ 3126-3136. (doi:10.1158/0008-5472.CAN-05-0425)

Yi M, Horton JD, Cohen JC, Hobbs HH \& Stephens RM 2006 WholePathwayScope: a comprehensive pathway-based analysis tool for high-throughput data. BMC Bioinformatics 7 30. (doi:10.1186/14712105-7-30)

Zamore PD \& Haley B 2005 Ribo-gnome: the big world of small RNAs. Science 309 1519-1524. (doi:10.1126/science.1111444)

Zhou Y, Zhu YZ, Zhang SH, Wang HM, Wang SY \& Yang XK 2011 MicroRNA expression profiles in premature ovarian failure patients and its potential regulate functions. Chinese Journal of Birth Health and Heredity 19 20-22.

Received 5 October 2011

First decision 21 November 2011

Revised manuscript received 14 May 2012

Accepted 30 May 2012 